\title{
BENEFÍCIOS DA FISIOTERAPIA MOTORA EM PACIENTES COM ESCLEROSE LATERAL AMIOTRÓFICA
}

\section{BENEFITS OF MOTOR PHYSICAL THERAPY IN PATIENTS WITH AMYOTROPHIC LATERAL SCLEROSIS}

Franciele Pinto da Silva Ramos ${ }^{1}$, Nillva Louzeiro de Almeida ${ }^{1}$; Aktor Hugo Teixeira²; Marcella Soares Carreiro Sales ${ }^{3}$.

RESUMO

Introdução: A Esclerose Lateral Amiotrófica (ELA) é uma doença degenerativa progressiva que lesa os neurônios motores em todos os níveis. A fisioterapia através de suas intervenções é parte da equipe de profissões envolvidas no tratamento da ELA. Objetivo: Verificar os tipos de condutas fisioterapêuticas utilizadas para o tratamento motor de pacientes com ELA e auxiliar fisioterapeutas no planejamento de condutas voltadas para essa de patologia. Material e Método: Foi realizada uma revisão da literatura nas bases de dados SCIELO, BVS, Medline, BIREME e literatura do acervo da biblioteca do Centro Universitário Unirg Gurupi/TO. Resultados: A realização de um programa de fisioterapia motora com uso de exercícios de fortalecimento e exercícios cinesioterapêuticos pode ser significativo no tratamento da ELA. Conclusão: A prática regular de exercícios de carga e intensidade moderadas pode resultar em melhora do déficit motor, da capacidade funcional e qualidade de vida do paciente com ELA. Todavia, não se tem resultados relacionados ao uso de recursos de correntes elétricas que promovem a contração muscular ativa. Os exercícios cinesioterapêuticos passivos promovem mobilidade e flexibilidade por meio de ações focadas em articulações e tecidos moles. Sua principal finalidade é a manutenção ou desenvolvimento do movimento livre e seus efeitos baseiam-se na melhora ou manutenção da força, resistência à fadiga, mobilidade, flexibilidade, relaxamento e coordenação motora. Observa-se ainda, que há poucos recursos terapêuticos a serem utilizados no tratamento da ELA e que entre os autores não há consenso quanto à melhor intervenção fisioterapêutica.

Descritores: Esclerose lateral amiotrófica; força muscular; fisioterapia. \begin{abstract}
Introduction: The amyotrophic lateral sclerosis (ALS) is a degenerative and progressive disease that damages motor neurons at all levels. Physiotherapy through their interventions is part of staff involved in the treatment of ALS. Objective: To verify the types of physiotherapeutic conduits used for the motor treatment of patients with ALS and to assist physiotherapists in the planning of conduits directed to this pathology. Material and Methods: A literature review was done in the databases SCIELO, BVS, Medline, BIREME and literature from the collection of the UnirG University Center in Gurupi City - TO. Results: The performance of physiotherapeutic program with the use of strengthening exercises and kinesiotherapeutic exercises may be significant in the treatment of ALS. Conclusion: The frequent practice of moderate loading and intensity exercises may result in improvement of the motor deficit, functional capacity and quality of life of the patient with ALS. However, there are no results related to the use of electric current resources that promote active muscle contraction. Passive kinesiotherapeutic exercises promote mobility and flexibility through actions focused on joints and soft tissues. Its main purpose is the maintenance or development of free movement and its effects are based on the improvement or maintenance of strength, resistance to fatigue, mobility, flexibility, relaxation and motor coordination. It is also observed that there are few therapeutic resources to be used in the treatment of ALS and that among the authors there is no consensus regarding the best physiotherapeutic intervention.
\end{abstract}

Keywords: Amyotrophic lateral sclerosis; Muscle strength; Physiotherapy.

\section{INTRODUÇAO}

A Esclerose Lateral Amiotrófica (ELA) é uma doença degenerativa progressiva que causa lesões nos neurônios motores em todos os níveis. É caracterizada por fraqueza muscular que aos poucos vai levando o indivíduo a um estado totalmente incapacitante, tornando-o assim

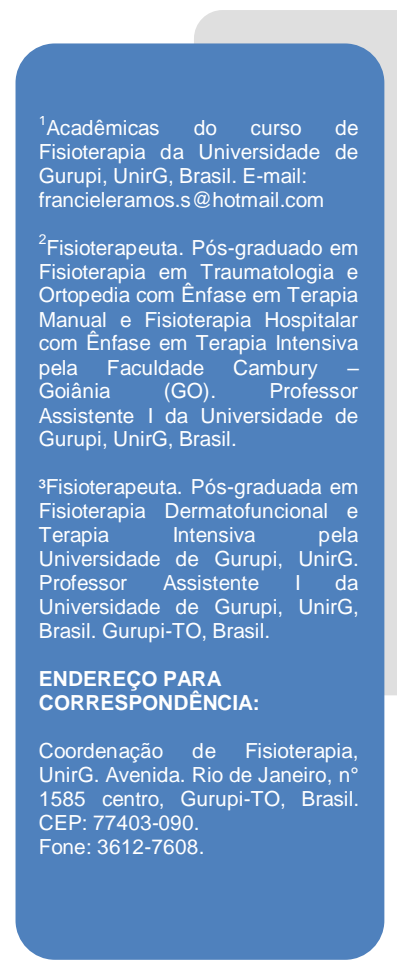

descondicionado fisicamente e impossibilitado de realizar suas atividades de vida diária ${ }^{1}$.

As raízes nervosas mais afetadas pela ELA são as raízes de C8-T1 em membros superiores (MMSS) e L5-S1 membros inferiores (MMII), desenvolvendo as dificuldades da coordenação motora fina, como movimentos de pinça e de tropeços devido à dificuldade de dorsiflexão do pé. 
Recebido: 30 maio 2017. Aceito: 11 dezembro 2018. Revista Amazônia Science \& Health - 2018 Out/Dez
Ramos, FPS. Almeida, NL. Teixeira, AH. Sales, MSC.

BENEFÍCIOS DA FISIOTERAPIA MOTORA EM PACIENTES COM ESCLEROSE LATERAL AMIOTRÓFICA.
A progressão da ELA acontece quando a medula espinhal mostra uma grave degeneração e perda de células do corno anterior em todos os níveis, há degeneração tanto do feixe corticoespinhal lateral como no anterior, sendo observada uma palidez na coloração da mielina ${ }^{2}$.

A evolução desta patologia é lenta e inicialmente, ocorre afecção apenas de um hemicorpo. Inicia-se nas extremidades, especialmente nos membros superiores progredindo para as demais áreas do corpo, o que pode fazer com que o indivíduo fique muito tempo imóvel ou acamado, levando assim a uma piora no déficit de força e atrofia muscular ${ }^{3-4}$.

Os pacientes com ELA apresentam frequentemente sinais de fraqueza muscular $(70 \%$ dos casos), atrofia (78\%) e queixas bulbares (22\%), sendo observados casos de demência associados em apenas $2 \%$ deles. Os sentidos (visão, audição, paladar, olfato e tato), assim como as capacidades mentais e psíquicas, não são afetados. $E$, na maioria dos casos, as funções sexuais, intestinal e visceral também não estão alteradas ${ }^{5}$

Ferraresso ${ }^{1,6}$ refere que a falência respiratória nestes pacientes é considerada a principal causa de morte. A evolução da doença resulta na incapacidade da ventilação normal dos pulmões. O comprometimento da musculatura inspiratória leva a insuficiência respiratória, hipoventilação alveolar e comprometimento da musculatura expiratória.

A diminuição da força, por sua vez, pode levar a deformidades articulares, dor, diminuição da amplitude de movimento (ADM), contraturas e rigidez. A fraqueza muscular progressiva é uma das maiores queixas de desconforto nestes pacientes, podendo assim diminuir a tolerância ao exercício físico e consequentemente aumento da fadiga ${ }^{7}$.

O fisioterapeuta como parte da equipe multidisciplinar desempenha um papel relevante no tratamento de pacientes com diagnóstico de ELA, pois através de métodos, técnicas e manobras fisioterapêuticas pode-se tratar os problemas que ocorrem no decorrer da progressão da doença ${ }^{8,3}$.

Cascon et al. ${ }^{9}$, afirma que, a prática de exercícios não aumenta a expectativa de vida, mas influencia na melhora da qualidade desta, no aprimoramento da funcionalidade física e na redução de incapacidades, contribuindo para uma melhora no estado de saúde do paciente.

Xerez $^{8,10}$ também afirma que a sobrevida média de pacientes com ELA é de 2 a 5 anos, mas que existem pacientes com mais de uma década. Que, no entanto, para alcançarem esta sobrevida, necessitam de uma assistência longa e criteriosa. A incidência da ELA na população mundial é relativamente uniforme e estão entre 1,5 e 2,5 casos por 100.000 habitantes por ano.

Em um estudo epidemiológico brasileiro, foram encontrados 433 pacientes com ELA, sendo
$58.5 \%$ do sexo masculino, com maior incidência na faixa etária de 65 a 74 anos. A idade média de início da doença foi de 52 anos. Sessenta e nove por cento eram pacientes com indício apendicular. A ELA familiar esteve presente em 5,9\% dos casos $^{11,12}$

É seguro afirmar que ainda não existe uma etiologia para a ELA, porém alguns fatores de risco têm sido citados como suspeitos para o seu desenvolvimento. Esses fatores incluem traumas, atividade física em excesso, dieta, excesso do consumo de vitamina $E$, participação em esportes de alto desempenho, residência em áreas rurais, consumo de álcool e cigarro, trabalhadores de indústrias metalúrgicas e com exposição e trauma elétrico ${ }^{2}$

Mediante as grandes repercussões motoras que os pacientes com ELA apresentam, o objetivo do presente estudo foi verificar os tipos de condutas fisioterapêuticas utilizadas para o tratamento motor de pacientes com ELA e auxiliar fisioterapeutas no planejamento de condutas voltadas para essa de patologia.

\section{MATERIAL E METODOS}

O presente estudo trata-se de uma revisão de literatura. A busca do acervo de artigos foi realizada através das bases de dados eletrônicos: Scientific Electronic Library Online (SCIELO), Biblioteca Virtual em Saúde (BVS), Medical Literature Analysis and Retrieval System Online (Medline) e Biblioteca Regional de Medicina (BIREME). Além disso, foram pesquisados livros da biblioteca do Centro Universitário Unirg - Gurupi - TO. Os descritores utilizados foram: Esclerose lateral amiotrófica, força muscular, fisioterapia.

Para criar o acervo de artigos foram selecionados estudos com títulos e resumos que abordassem como tema ELA, sendo publicações no idioma inglês, português e espanhol e de domínio público (disponibilidade online).

Os critérios de inclusão foram: estudos que abordaram a eficácia de exercícios de fortalecimento muscular e condutas fisioterapêuticas utilizadas em pacientes com ELA, publicados entre os anos de 2000 a 2016 . Foram excluídas literaturas e publicações que não estivessem de acordo com os objetivos da revisão. Ao final, pesquisa foi embasada em 22 artigos que cumpriram os quesitos pré-estabelecidos.

O presente estudo por ser de revisão, não foi submetido à avaliação do Comitê de Ética em Pesquisa, porém todos os preceitos éticos estabelecidos foram respeitados no que se refere a zelar pela legitimidade das informações. 
A ELA é uma doença que leva a uma perda progressiva de massa muscular e consequentemente ao enfraquecimento deste sistema. A fraqueza muscular e a fadiga são características relevantes no quadro clínico de pacientes com ELA. Como resultado leva o indivíduo a uma gradativa dependência e deficiência para realizar suas atividades de vida diária evoluindo aos estágios mais graves com insuficiência respiratória ${ }^{3,10}$.

Segundo Cascon et $\mathrm{al}^{9}$ nas doenças neuromusculares, desde que os exercícios resistidos moderados causem aumento da força dinâmica, isométrica e isocinética dos membros superiores e inferiores, os mesmos podem reduzir a sarcopenia, reduzir a dor, melhorar a mobilidade articular e função respiratória, contribuindo muito para a melhora da qualidade de vida do paciente.

O papel do fisioterapeuta não engloba apenas - caráter reabilitativo. O mesmo deve realizar orientações de cunho educativo no que se refere a adaptações domiciliares. Visto que muitos problemas podem ser evitados ou diminuídos quando pacientes e pessoas envolvidas no cuidado recebem orientações precisas. Tais esclarecimentos podem evitar quedas, facilitar transferências e evitar aparição de lesões de pressão ${ }^{8}$.

O tratamento de pacientes com ELA é complexo e pode ser considerado um desafio. Por não se conhecer muito sobre a etiologia da doença, não existe um protocolo de tratamento específico para pacientes com esta patologia. Mas embora seja uma condição incurável até o momento, um apoio multidisciplinar pode melhorar a qualidade de vida e a sobrevivência desses indivíduos ${ }^{3,8}$.

Ainda não existe um consenso na literatura em relação à prescrição de exercícios para pessoas com ELA, devido à dificuldade em formular um protocolo eficaz e que não sobrecarregue o sistema muscular levando a lesões através da prática excessiva de atividades ou a realização de exercícios de fortalecimento. Mas têm-se evidências de que há efeitos fisiológicos e psicológicos positivos como resultados da prática, desde que sejam aplicados na fase inicial da doença ${ }^{13}$.

A fisioterapia motora tem como principal objetivo obter o maior grau possível de independência através da mobilidade funcional. $\mathrm{O}$ fisioterapeuta deve estar atento às individualidades de cada paciente, considerando o grau de força muscular dos membros superiores e inferiores, tônus musculares, o padrão de marcha e o gasto de energia que será necessário para a realização de cada atividade ${ }^{7}$.

Onze dos vinte e dois artigos avaliados para esta revisão ofereceram conclusões significativas no contexto de reabilitação, assistência fisioterapêutica e multifatorial e exercícios de fortalecimentos de caráter moderado e submáximos no paciente com ELA. Os mesmos compõem a tabela abaixo e fortalecem que a fisioterapia faz parte do processo de tratamento do paciente com ELA melhorando a qualidade de vida.

Tabela 1. Autores, ano de publicação e resultados dos artigos incluídos nesta revisão.

\begin{tabular}{|c|c|c|}
\hline AUTOR & $\begin{array}{c}\text { ANO DE } \\
\text { PUBLICAÇÃO }\end{array}$ & RESULTADOS \\
\hline $\begin{array}{l}\text { Calzada-Sierra, L. } \\
\text { Gómez-Fernández }\end{array}$ & 2001 & $\begin{array}{l}\text { Reabilitação multifatorial é a melhor esperança para melhorar a saúde e a } \\
\text { sobrevivência destes pacientes com ELA. Os resultados foram } \\
\text { significativos: Quatro dos seis pacientes apresentaram melhoria na sua } \\
\text { qualidade de vida com a intervenção precoce. }\end{array}$ \\
\hline Pozza AM, et al. & 2006 & $\begin{array}{l}\text { Os exercícios moderados e de forma regular trazem benefícios à } \\
\text { pacientes com ELA desde que sejam monitorados por profissionais } \\
\text { capacitados. }\end{array}$ \\
\hline $\begin{array}{l}\text { Elizabeth SH e Emerson } \\
\text { FM. }\end{array}$ & 2008 & $\begin{array}{l}\text { A assistência fisioterapêutica é caracterizada por três abordagens } \\
\text { principais: paliativa, motora e respiratória. Apesar de necessitarem ainda } \\
\text { de esclarecimentos para uma conduta motora mais ativa. Intervenções } \\
\text { com recrutamento neuromotor mais ativo possibilitam maiores efeitos para } \\
\text { o bem-estar e a qualidade de vida de pacientes com ELA. }\end{array}$ \\
\hline
\end{tabular}




\begin{tabular}{|c|c|c|}
\hline $\begin{array}{l}\text { Lívia DF, Marco O, ASD } \\
\text { de L. }\end{array}$ & 2009 & $\begin{array}{l}\text { A realização de um programa de exercícios de fortalecimento pode ser } \\
\text { significativa no tratamento. A prática regular de exercícios com carga e de } \\
\text { intensidade moderada, pode ter como resultado: a melhora do déficit } \\
\text { motor, capacidade funcional e da qualidade de vida. }\end{array}$ \\
\hline Orsini M et al. & 2009 & $\begin{array}{l}\text { O conhecimento dos estágios da doença pode auxiliar o fisioterapeuta na } \\
\text { determinação da conduta específica, desde que o profissional tenha } \\
\text { conhecimento para colocar o paciente no estagio correto. Relacionado ao } \\
\text { exercício de ganho de força o fisioterapeuta deve utilizar exercícios } \\
\text { submáximos com objetivo de retardar a perda de força muscular. }\end{array}$ \\
\hline $\begin{array}{l}\text { Facchinetti LD, Orsini M, } \\
\text { Lima MASD }\end{array}$ & 2009 & $\begin{array}{l}\text { Um programa de exercícios de fortalecimento pode ser um componente } \\
\text { essencial do tratamento; a prática regular de exercícios de carga e } \\
\text { intensidade moderadas pode resultar em melhora do déficit motor, da } \\
\text { capacidade funcional e da qualidade de vida de indivíduos com ELA. }\end{array}$ \\
\hline
\end{tabular}

\section{Cascon R, Orsini M et}

al. Silva NPO.
2010
A prática de exercícios embora não aumente a expectativa de vida dos pacientes até o momento, pode influenciar positivamente na qualidade de vida e no desempenho funcional desses indivíduos.

Apesar da literatura mostrar que os efeitos de exercícios moderados não são duradouros em pacientes com ELA, devem sim serem indicados pois a prática regular de tais exercícios contribuem para a melhora no aspecto motor, na capacidade funcional e na qualidade de vida destes pacientes.

Várias estratégias têm colaborado para a melhora da qualidade de vida

Eduardo LJ

2013 dos indivíduos com ELA. E dentre as abordagens, a fisioterapia tem um papel muito importante.

A fadiga muscular deve ser primeiramente avaliada antes de se indicar

Martins LJNS et al.

2013 uma conduta terapêutica a fim de se alcançar os benefícios desejados na ELA.

\begin{tabular}{|c|c|c|}
\hline Guimarães MTS, Vale & 2016 & $\begin{array}{l}\text { Mesmo não impedindo o curso natural da doença, a fisioterapia } \\
\text { neurofuncional mostrou trazer importantes benefícios para os pacientes. }\end{array}$ \\
\hline
\end{tabular}

Existem muitas dúvidas sobre a recomendação ou não de exercícios regulares e outros recursos fisioterapêuticos para pacientes com ELA. Dentre as intervenções que podem ser consideradas benéficas está a fisioterapia motora, que pode promover a melhora da qualidade de vida por meio de programas de tratamento, para a melhora funcional do paciente ${ }^{14}$.

A fisioterapia tem um papel importante no tratamento de pacientes com esclerose lateral amiotrófica, pois muitas complicações que aparecem no decorrer da doença podem ser tratadas por métodos e técnicas da fisioterapia 8,15

No entanto, existem dois fatores que devem ser considerados antes de planejar ou programar uma atividade ou um exercício para pacientes com ELA, que são: a atrofia por desuso e prevenção por uso excessivo. É papel do fisioterapeuta monitorar os exercícios e atividades específicas, para evitar que diminuição de força muscular ou fadiga ocorra por causa da prática destes $^{16}$.

Não se tem resultados relacionados ao uso de recursos de correntes elétricas que promovem a contração muscular ativa. Devido acreditarem que tais recursos podem acarretar na exacerbação do processo neurodegenerativo e acelerar a progressão da doença ${ }^{14}$.

Os exercícios cinesioterápicos passivos são bem destacáveis entre as principais condutas, pois promovem mobilidade e flexibilidade por meio de ações focadas em articulações e tecidos moles. Em alguns estudos 
Recebido: 30 maio 2017. Aceito: 11 dezembro 2018. Revista Amazônia Science \& Health - 2018 Out/Dez
Ramos, FPS. Almeida, NL. Teixeira, AH. Sales, MSC.

BENEFÍCIOS DA FISIOTERAPIA MOTORA EM PACIENTES COM ESCLEROSE LATERAL AMIOTRÓFICA. já foi possível observar melhora da qualidade de vida com a fisioterapia moderada regular ${ }^{8}$.

De acordo com Júnior, um músculo fraco pode ser lesionado devido o excesso de trabalho, e por isso, exercícios físicos resistidos para pacientes com ELA, devem ser de resistência moderada e cuidadosamente recomendado por um fisioterapeuta, pois são benéficos e ajudam na manutenção da função ${ }^{8}$.

É comum o aparecimento de alterações musculoesqueléticas em indivíduos portadores dessa patologia, como encurtamentos, contraturas e uma postura, ocorrido devido a perda progressiva de força muscular. Assim, alongamentos e exercícios, que visam a melhora da flexibilidade, podem prevenir estas alterações ${ }^{8 .}$

O exercício terapêutico tem como objetivo manter, corrigir e/ou recuperar uma determinada função, ou seja, restaurar a função normal de corpo ou manter o bem-estar. Sua principal finalidade é a manutenção ou desenvolvimento do movimento livre para a sua função, e seus efeitos baseiam-se no desenvolvimento, melhora, restauração e manutenção da força, da resistência à fadiga, da mobilidade e flexibilidade, do relaxamento e da coordenação motora ${ }^{15-19}$

Gómez Fernández e Calzada Sierra ${ }^{\text {i7-18, }}$ realizaram um estudo com um programa de reabilitação a fim de manter a capacidade funcional e prevenir complicações da imobilidade muscular. Utilizando cinesioterapia, tratamento respiratório e motor, por meio de exercícios de alongamento e fortalecimento muscular, reeducação postural, e outros exercícios, bem como técnicas para relaxamento. Tiveram resultados satisfatórios, pois 4 dos 6 voluntários do estudo apresentaram melhora da qualidade de vida.

Porém no estudo de Pedroso et $\mathrm{al}^{17-18,21}$, os resultados não foram os mesmos. Foram realizadas intervenções fisioterapêuticas por um período de seis meses, com condutas de exercícios respiratórios e fisioterapia motora. Avaliaram dois grupos, sendo um grupo controle, e outro recebendo intervenções fisioterapêuticas. Ao final, não foram observados qualquer tipo de evolução em ambos os grupos.

\section{CONCLUSAOO}

A partir desta revisão bibliográfica, concluise que a fisioterapia motora tem um papel muito importante no tratamento de pacientes com ELA, pois apesar de ainda necessitarem de mais estudos de casos que comprovem sua eficácia, muitos autores afirmam que a fisioterapia através da prática regular de exercícios e de condutas que envolvem alongamentos, mobilização, reeducação postural e exercícios ativos, principalmente os de prática regular com carga e intensidade moderadas, podem contribuir para a melhora do déficit motor e qualidade de vida. Não se tem ainda resultados conclusivos sobre a utilização de correntes elétricas visto que acredita-se que tal conduta pode acarretar na exacerbação do processo neurodegenerativo e acelerar a progressão da doença.

Por ainda não existir um protocolo específico que oriente 0 fisioterapeuta na elaboração de seu plano de tratamento para estes tipos de pacientes, o profissional deve estar atento às particularidades de cada paciente, e ter total conhecimento do estágio da doença em que o indivíduo se encontra.

\section{REFERÉNCIAS}

1 Ferraresso A. Avaliação clínica e funcional do comprometimento respiratório de pacientes com esclerose lateral amiotrófica. Dissertação de mestrado em Ciências na área de concentração Clínica Médica, Faculdade de Ciências Médicas da Universidade Estadual de Campinas. Campinas-SP. 2013.

2 Neto JPB, Takayanagui OM., Tratado de Neurologia da Academia Brasileira de Neurologia. Ed 1. Elsevier. 2013.

3 Martins LJNS, Silva NPO, Ferreira TB, Cavalcanti FAC. Associação entre fadiga e fatores clínico-pessoais de pacientes com esclerose lateral amiotrófica. ConScientiae Saúde.2013;12(4):604-10.

4 Bandeira FM, Quadros NNCL, Almeida KJQ, Caldeira RM. Avaliação da qualidade de vida de pacientes portadores de Esclerose Lateral Amiotrófica (ELA) em Brasília. Rev Neurocienc. 2010;18(2):133-38.

5 Fukujima MM; et al. Atualização em fisioterapia na emergência: Atendimentos a pacientes com Esclerose Lateral Amiotrófica. São Paulo - SP: Unifesp. p 56. 2009.

6 Presto B; et al. Ventilação Não-Invasiva e Fisioterapia Respiratória para pacientes com Esclerose Lateral Amiotrófica. Rev Neurocienc 2009;17(3): 293-7.

7 Facchinetti LD, Orsini M, Lima MASD. Os riscos do exercício excessivo na esclerose lateral amiotrófica: atualização da literatura. Rev Bras Neurol. 2009;45(3);33-8. 
8 Júnior EL. Abordagem Fisioterapêutica na Esclerose Lateral Amiotrófica: artigo de atualização. Rev Neurocienc 2013;21(2);313-318.

9 Cascon R, Orsini M, Leite MA, Freitas MRG, Nascimento OJM. The resistance exercise in Amyotrophic Lateral Sclerosis (ALS): update. Rev Neurociênc. 2010;18(1):116-21.

10 Xerez DR. Reabilitação na Esclerose Lateral Amiotrófica: revisão da literatura. Acta Fisiatr 2008; 15(3):182-88.

11 Dietrich FN, et al. Esclerose Lateral Amiotrófica NO BRASIL. Arquivos de NeuroPsiquiatria. São Paulo. 2013;58(3):607-615.

12 Fga SP, Lima JMB, Alvarenga RP. Epidemiologia da Esclerose Lateral AmiotróficaEuropa/América do Norte/América do Sul/Ásia.Discrepâncias e similaridades. Revisão sistemática da literatura. Rev Bras Neurol. 2009;45 (2): 5-10.

13 Costa FA; Martins LJNS, Silva NPO. A Esclerose Lateral Amiotrófica e a Fisioterapia Motora. 2011.Monografia (trabalho de conclusão de curso) Universidade Federal do Rio Grande do Norte - UFRN.

14 Honorato ES, Martins EF. Evidências para direcionamento da intervenção fisioterapêutica nas alterações cinético-funcionais geradas pela Esclerose Lateral Amiotrófica. Rev Atenção Saúde. 2008;6(15):66-74.

15 Guimarães MTS, Vale VD, Aoki T. Os benefícios da fisioterapia neurofuncional em pacientes com Esclerose Lateral Amiotrófica: revisão sistemática. ABCS Health Sci. 2016; 41(2):84-89.

16 Orsini M, Freitas MRG, Mello MP, Antonioli RS, JPB Reis, Nascimento OJM, et al. Reabilitação física na Esclerose Lateral. Rev Neurocienc. $\quad 2009 ; \quad$ 17(1):31-36.

17 Gómez FL, Calzada SDJ. The importance ofmultifactorial rehabilitation treatment in amyotrophic lateral sclerosis. Rev Neuro. 2001;32(5):423-6 .

18 Pedroso JPC, Ramirez C, Silva HCA, et al. Importance of the physical therapeutic program in muscular force and the functional state in patients with amyotrophic lateral sclerosis during 6 months. Proceedings of the 12th International Symposium on ALS/MND; 2001;1820;Oakland,USA;2001.p.94.
19 Orsini M, Freitas MRG, Mello M P, Botelho JP , Cardoso FM, Nascimento OJM, FreitasGR6. Medidas de Avaliação na Esclerose Lateral Amiotrófica. Revista Neurociências. Niterói, RJ. 2008;16( 2): 144-151.

20 Lima NMFV, Guerra CC, Teixeira LC, Silva LBC, Sordi M, Mourão L, et.al. Tradução e validação da versão brasileira da escala de gravidade na esclerose lateral amiotrófica (Egela) Fisioterapia e pesquisa. 2009;16(4):316-22.

21 Pozza AM, Delamura MK, Ramirez C, Valério NI, Marino LHC, Lamari NM. Physiotherapeutic conduct in amyotrophic lateral sclerosis. Sao Paulo Med J. 2006; 124(6):350-4. 